\title{
Auditory evoked brain responses: Intensity functions from bipolar human scalp recordings*
}

\author{
DONALD I. TEPASt, LAWRENCE A. BOXERMAN, and A. MICHAEL ANCH
} Saint Louis University, St. Louis, Missouri 63103

Average evoked brain responses were computed from four simultaneous recordings during binaural tone stimulation at 10 intensity levels. Amplitudes and latencies were measured for the prominent components, and linear regression coefficients were computed. The results indicate that the sensitivity of the evoked response to stimulus intensity is dependent upon the selection of the appropriate measure. Linear amplitude functions were demonstrated. Additional research relating these responses to psychophysical measures is suggested.

Numerous studies have examined the relationship of the averaged evoked brain response (EBR) to the intensity of a tone. One approach to relating EBR measures to sensation compares EBR detection thresholds with psychophysical absolute thresholds. These studies have met with a remarkable but limited degree of success (Price, 1969). This is not surprising, given the problems of psychophysical threshold measurement and scalp recording (Donchin \& Sutton, 1970). Another approach is to relate EBR measures to psychophysical measures of subjective magnitude. Buchsbaum (1971) has discounted this approach by questioning the generality of studies which show EBR amplitude to increase with stimulus intensity. The study reported here is an initial effort to clarify the EBR amplitude-intensity relationship as a preliminary to direct comparisons of the EBR to subjective magnitude measures.

In the study reported here, the magnitude of the auditory stimulus is varied in small 2-dB steps over a limited range to determine if EBR measures of stimulus change have a sensitivity comparable to psychophysical measures. In addition, the responses were recorded from experienced Ss using methodological controls appropriate for comparable psychophysical studies. Finally, simultaneous recordings were obtained from several scalp electrodes to assess the relative sensitivity of a number of EBR measures.

\footnotetext{
* This research was supported by Research Grant MH 14020-03 from the National Institute of Mental Health. A portion of the data of this study was reported at the Psychonomic Society meetings, San Antonio, Texas, November 1970.

tAddress: Department of Psychology, Saint Louis University, 221 N. Grand Blvd.,
} St. Louis, Missouri 63103 . converter at the $1 / \mathrm{sec}$ rate. Additional Series 1200 modules, as shown in Fig. 1, controlled the output of a Hewlett-Packard Model HP3300A function generator so that $1 \cdot \mathrm{kHz}$ signals, $30 \mathrm{msec}$ long with a $10-\mathrm{msec}$ rise-fall time, were delivered to matched TDH39 (Telephonics) earphones at appropriate levels. These earphones were mounted in MX41/AR cushions and were worn by the $S$ with an HD11B headset. Acoustical calibration was performed at the earphones using a Bruel and Kjaer Model 158 audiometer calibrator with an NBS 9A 6 cc acoustic coupler.

The electroencephalogram (EEG) was recorded with Grass E5S electrodes, fastened to the scalp with collodion, at Positions $\mathbf{F z}, \mathbf{C z}, \mathbf{P z}$, and $\mathrm{Oz}$, as designated by the international electrode method (Jasper, 1958). A Beckman $16 \cdot \mathrm{mm}$ biopotential electrode, attached to the forehead with a two-sided adhesive collar, served as the ground. The EEG potentials were amplified and continuously recorded by a Grass Model 7 polygraph. Amplified potentials from the driver amplifiers of the polygraph were then distributed to the AX08 analog inputs of the LAB-8 computer system. The minimum time constant used was $0.4 \mathrm{sec}$, and the high-frequency response was limited to $0.5 \mathrm{kHz}$ for all analog inputs to the AX08.

EBRs were computed by the Digital Equipment Corporation LAB- 8 computer system using the advanced averager program (DEC-LB-U18C-PB). Four channels were simultaneously averaged. For each channel, computations were made at 200 time points distributed over a $398-\mathrm{msec}$ analysis time. Each EBR was the sum of 240 EEG responses. A 100-msec square wave output of a Grass SD5 the LAB-8 computer system and a
Grason-Stadler Series 1200 input pulse generator at this rate. The output of this pulse generator triggered both the AX08 sync input of

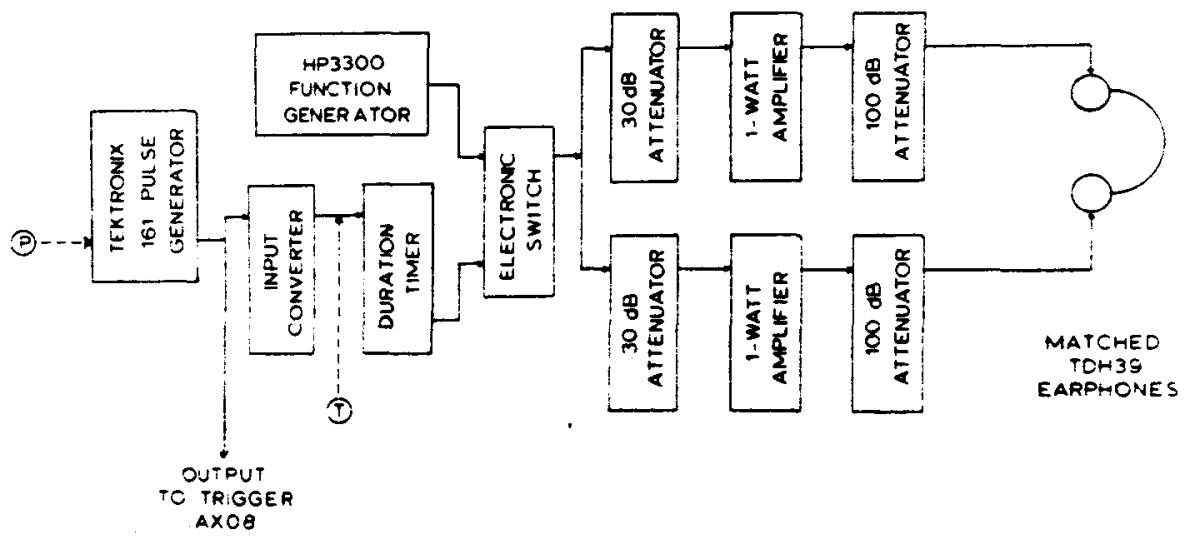

BINAURAL STIMULATION SYSTEM

Fig. 1. The binaural stimulation system. 
TD-E $1000 \mathrm{HZ}$ Y/SECOND N-240 $67 \mathrm{dE}$

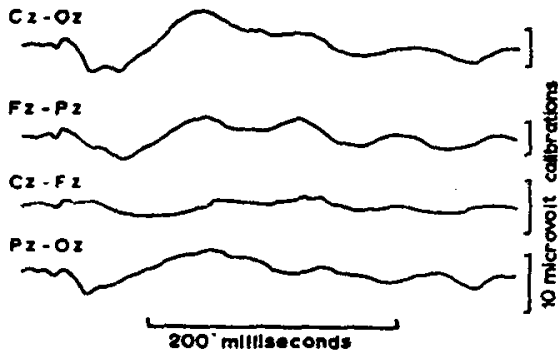

Fig. 2. Four simultaneously computed bipolar evoked brain response waveforms recorded from $\mathbf{S} \mathbf{T D}$ in Sersion 6.

stimulator reduced to the 10 -microV level was used to calibrate the EBR measurements. This calibration signal was averaged in a manner identical to that used for the S's EEG at the beginning and end of every session.

\section{Procedure}

Each $S$ was tested on 10 occasions. Responses to 10 intensity levels were obtained during each of these sessions. Intensity was varied in 2-dB steps over a 20-dB range, with the highest intensity being $95 \mathrm{~dB}$ SPL re 0.0002 dyne/c $\mathrm{cm}^{2}$. The order of presentation for the various stimulus conditions was varied in a preset random manner for the 10 trials of each session in an effort to control for possible order effects. A different random sequence was used for each session, and the $S 6$ were not provided with any information from $E$ as to the order of presentation. Thus, each of the 10 stimulus intensities was presented to each $S$ once in each of the 10 experimental sessions.

For the trials, the Ss were simply instructed to listen to the tones and to minimize bedy movements and other behaviors which might produce noise in the recordings. The Ss were comfortably seated in the dark on an adjustable dental chair within a shielded experimental chamber. E indicated the beginning and end of each trial to $\mathbf{S}$ by flashing a small red indicator light in the chamber. Averaging was not begun until the $\mathbf{S}$ signaled he was ready by closing a hand-held pushbutton and until $E$ judged the EEG polygraph recording to be artifact free. The time between trials was a constant 4-min period during which the $S$ was free to exercise any bodily movements he wished, given the confines of the chair and electrode leads.

EBRs were simultaneously computed from four bipolar electrode combinations: $\mathrm{Cz}-\mathrm{Oz}, \mathrm{Fz}-\mathrm{Pz}, \mathrm{Cz}-\mathrm{Fz}$, and $\mathrm{Pz}-\mathrm{Oz}$. These averages were computed in real time during the trials, and the resulting averages were photographed or plotted by $\mathrm{E}$ during the between-trial period for later manual measurement of the responses. At the end of each session, the electrodes were removed from $S$, and then $E$ scheduled $S$ for his next session at the same time of day. Manual measurement of the EBRs by $E$ was undertaken when the $S$ had completed all 10 sessions.

\section{RESULTS}

Figure 2 shows an example of the EBR waveforms obtained in this study. In this figure, the four bipolar EBR waveforms recorded from one $S$ (T.D.) on one trial in Session 6 are presented. For this $\mathrm{S}$, the same major features are evident in varying degree within all response waveforms. In Fig. 3, all of the $\mathrm{Cz}-\mathrm{Oz}$ waveforms obtained from the same $S$ (T.D.) in Session 4 are presented. Similar response components are evident to a varying degree at all levels of stimulus magnitude. These response components are labeled in Fig. 4 for purposes of identification and description. 1 This waveform was recorded from T.D. in Session 5 . Since the waveforms shown in these three figures were recorded from the same $S$ in different sessions, a crude indication of the consistency of the data can be obtained by comparing these figures.

In general, the components were most evident and easily identified in the $\mathrm{Cz}-\mathrm{Oz}$ waveform. For two of the Ss (T.D. and J.N.), these features were identifiable in all four bipolar waveforms, and the following measurements were made on all of their waveforms: Amplitudes $A$ to $B$, $B$ to $C$, and $C$ to $D$; peak latencies of $A, B, C$, and D. For the third S (J.B.), reliable manual measurements could be made only on the $\mathrm{Cz}-\mathrm{Oz}$ waveform

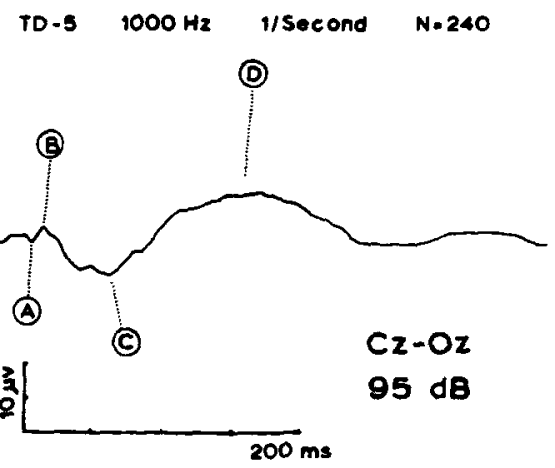

Fig. 4. The $\mathrm{Cz}-\mathrm{Oz}$ evoked brain response waveform recorded from S T.D. on one trial in Session 5 . The response components measured are identified alphabetically in order of occurrence.
TD-4 $1000 \mathrm{~Hz}-1 /$ sec.

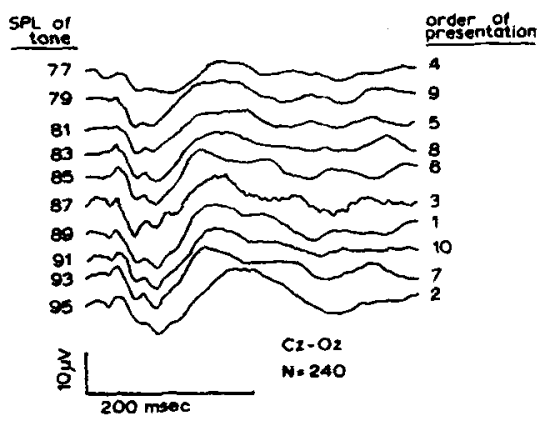

Fig. 3. All $\mathrm{Cz}-\mathrm{Oz}$ evoked brair response waveforms recorded from S T.D. in Sescion 4. Downward deflection indicates that the $O$ electrode was positive with respect to the $\mathrm{Cz}$ electrode.

amplitudes. Means were computed for each of the S-treatment-measure combinations studied. Linear regression lines were then fitted to the data by least-squares computation and correlation coefficients determined by the LAB-8 computer system using the general linear regression program (DECUS No. 8-118). Regression lines, together with the mean data points, were then graphed for all seven measures on each of the four waveforms for two of the $\mathrm{Ss}$ and for the three amplitude measures on one waveform for the third S.

Correlation coefficients for the EBR amplitude measurements from T.D. and J.N. are presented in Table 1. High statistically significant correlations between $\mathrm{Cz}-\mathrm{Oz}$ amplitude measures and SPL were revealed for both of these Ss. The regression lines and mean data points for the $\mathrm{Cz}-\mathrm{Oz}$ amplitude measures from these two $S$ s are graphically presented in Fig. 5. For J.B., the coefficients were $.78, .85$, and .25 for, respectively, the A-B, B-C, and $\mathrm{C}-\mathrm{D} \mathrm{Cz}-\mathrm{Oz}$ amplitude measures. Thus, a statistically significant positive correlation between $\mathrm{Cz}_{-}-\mathrm{Oz}$ B-C amplitude and SPL was obtained from all three Ss.

Given the results of the analysis of the amplitude data from the individual Ss, the $\mathrm{Cr}_{2}-\mathrm{Oz}_{2}$ amplitude meamures from all three Ss were pooled together. The mean data points and regrescion lines for the pooled data are shown in Fig. 6. For these data the correlation coefficients were $.64, .97$, and .96 for, respectively, the A-B, B-C, and C-D $\mathrm{Cz}-\mathrm{Oz}$ amplitude messures. Thus, the regression lines fitted to the pooled $B-C$ and $C-D$ mean amplitudes accounted for over $92 \%$ of the variance.

Table 2 shows the correlation coefficients for the EBR latency measurements from T.D. and J.N. for 
Table 1

Correlations of EBR Amplitude Measures with SPL for Ss TD and JN

\begin{tabular}{cccccccc}
\hline $\begin{array}{c}\text { Bipolar } \\
\text { Electrode } \\
\text { Combination }\end{array}$ & A-B & B-C & C-D & & \multicolumn{3}{c}{ S JN } \\
\hline Cz-Oz & -.20 & $.92 \dagger$ & $.96 \dagger$ & & $.94 \dagger$ & $.90 \dagger$ & $.93 \dagger$ \\
Fz-Pz & -.03 & $.87 \dagger$ & $.93 \dagger$ & & -.56 & $-.64 *$ & $.67 *$ \\
Cz-Fz & -.45 & .28 & $.75 *$ & & .42 & .46 & -.44 \\
Pz-Oz & -.13 & -.50 & $.74 *$ & -.56 & -.25 & $.84 \dagger$ \\
\hline
\end{tabular}

$* p<.05,+p<.01$

all four bipolar recordings. Although high, statistically significant correlations between latency measures and SPL were revealed for both $\mathrm{S} s$, it is difficult to note clearly high agreement between Ss. Figure 7 shows the regression lines and mean data points for the $\mathrm{Cz}-\mathrm{Oz}$ latency measures from J.N. Similar to amplitude data, $\mathrm{Cz}-\mathrm{Oz}$ latency measures are correlated
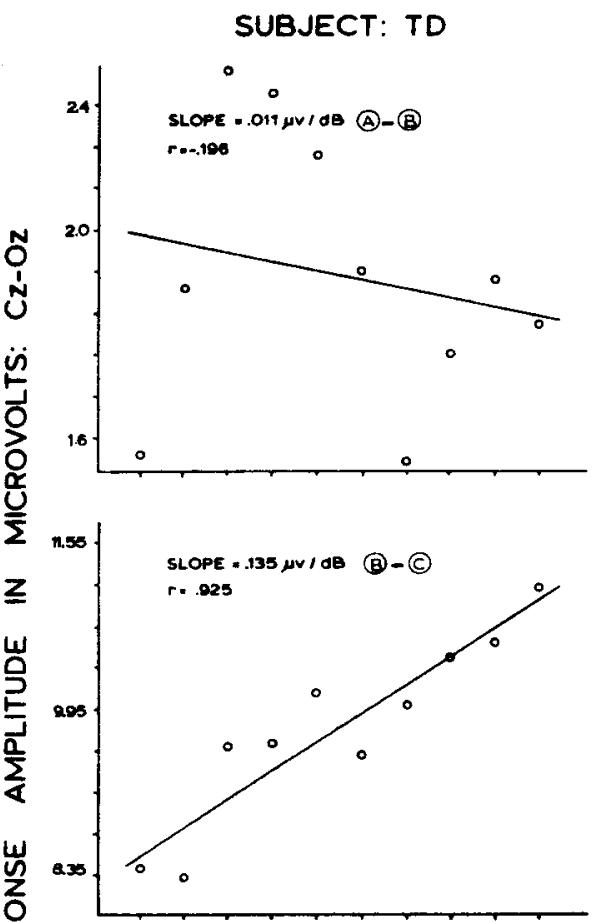

with SPL. Unlike amplitude data, the highest correlations are not necessarily obtained with the $\mathrm{Cz}-\mathrm{Oz}$ latency measures. Data were not pooled across Ss, since data from only two $S s$ were available and agreement between $S_{8}$ was not clear.

\section{DISCUSSION}

The data clearly show that the amplitude of the auditory EBR
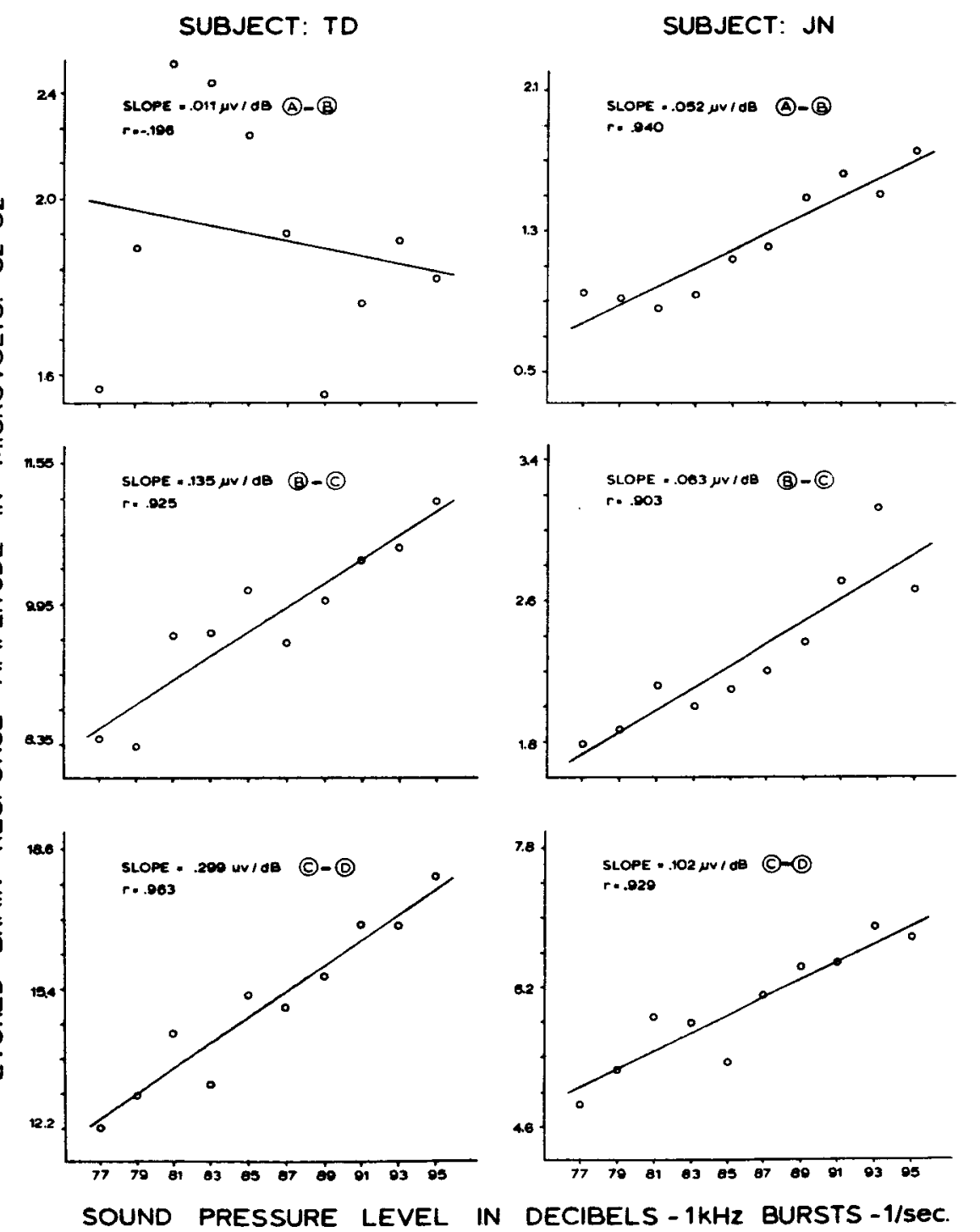

Fig. 5. Evoked brain response amplitude-intensity functions for Ss T.D. and J.N. The regression lines shown were fit to these mean data points using the method of least squares.
POOLED DATA

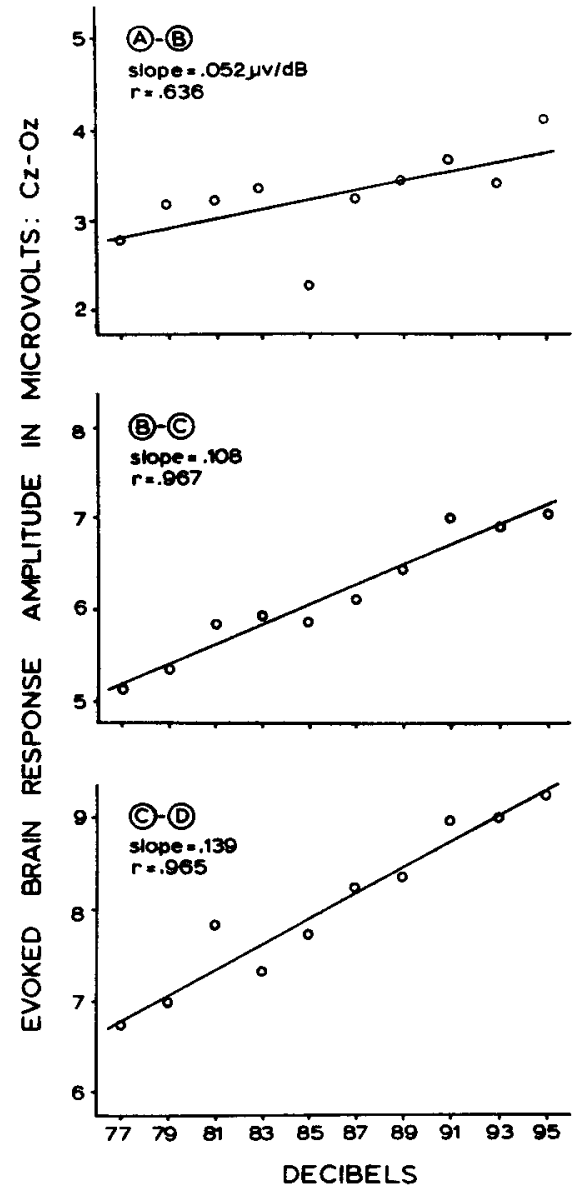

Fig. 6. Pooled evoked brain response amplitude-intensity functions for So T.D., J.N., and J.B. The regression lines shown were fit to the mean data points obtained when the data from all three Ss were grouped together.

increases as a function of the intensity of the stimulus. Since the auditory stimulus was manipulated in small steps over a limited range, there is reason to suggest that the sensitivity of EBR measures may approach that of psychophysical determinations. Similar conclusions have been drawn for the visual EBR (Siegfried et al, 1965). These results indicate that the sensitivity of the EBR to stimulus intensity is dependent upon the selection of an appropriate waveform measure. Insignificant correlations and negative relationships can be obtained if inappropriate components or potentials are studied. Our study has incorporated a number of methodological strategies which we feel are essential to this demonstration: averaging of a large number of EEG responses $(N=240)$ in the computation of each EBR; repeated testing of experienced Ss (10 
Table 2

Correlation of EBR Latency Measures with SPL for Ss TD and JN

Bipolar

Electrode

Combi-

S TD

\begin{tabular}{lcccccccc} 
nation & A & B & C & D & A & B & C & D \\
\hline $\mathrm{Cz}-\mathrm{Oz}$ & $-.92 \dagger$ & $-.73^{*}$ & .18 & .13 & $-.72^{*}$ & $-.88 \dagger$ & $-.90 \dagger$ & $-.81 \dagger$ \\
$\mathrm{Fz}-\mathrm{Pz}$ & $-.95 \dagger$ & $-.84 \dagger$ & -.50 & -.55 & $.68 *$ & .39 & -.46 & $-.81 \dagger$ \\
$\mathrm{Cz}-\mathrm{Fz}$ & $-.81 \dagger$ & $-.69^{*}$ & -.22 & -.01 & .22 & $.82 \dagger$ & .63 & $-.88 \dagger$ \\
$\mathrm{Pz}-\mathrm{Oz}$ & $-.91 \dagger$ & $--.66^{*}$ & -.01 & .37 & -.18 & $-.72^{*}$ & $-.77 \dagger$ & $-.81 \dagger$ \\
\hline
\end{tabular}

$*_{p}<.05,+p<.01$

sessions) with order effects controlled; administration of all stimulus conditions during every session; selection of appropriate stimulus conditions; and precise control of all stimulus parameters. Many of these methodological strategies parallel those of traditional psychophysics.

We suggest that these methodological strategies, together with the selection of the appropriate EBR measure, are required if the EBR is to be related to psychophysical measures of subjective magnitude. The failure of some studies to relate EBR magnitude in a reliable manner to stimulus intensity may very well be related to a failure to control appropriately some of these factors. Similarly, many studies which have related the $E B R$ to stimulus intensity have been disturbed by the variability of the data (Davis et al, 1967; Price, 1969). Most of these studies are primarily interested in the immediate development of a technique for clinical audiometry, and the conditions of testing appear to be limited to methods which might be practical in a clinical setting. Our study has made no attempt to impose such methodological restrictions. As a result, we have been impressed with the stability of our EBR intensity determinations. Research directly examining the variability of the auditory EBR intensity functions under a variety of conditions is in order.

The isolation of $\mathrm{Cz} \cdot \mathrm{Oz}$ as an appropriate bipolar placement for the study of the auditory EBR appears to be quite compatible with the monopolar study of the response distribution on the scalp reported by Vaughan and Ritter (1970). Similarly, the identification of $B-C$ and $C-D$ measures as sensitive indices of the intensity of the stimulus appears to be compatible with monopolar data (Rapin et al, 1966; Davis, 1968). Additional research comparing simultaneously recorded monopolar and bipolar auditory EBR intensity functions under appropriately controlled conditions is needed before the relative merits of monopolar and bipolar recording approaches can be evaluated. The failure to identify clearly latency measures which were sensitive indicants of stimulus intensity for all Ss tested may be related to several factors: inadequate temporal resolution (one data point per $2 \mathrm{msec}$ ) for the averaging program or the waveform photography used in the present study; failure to test additional $S$ s and other electrode combinations; or latency shifts associated with the specific number of EEG responses averaged in the present study (Shepherd, 1969). Perhaps the most likely suggestion is that latency is not a sensitive measure under these stimulus conditions. In any case, additional research is required before

ELECTRODE PLACEMENT: CZ to O2 SUBJECT: IN $1000 \mathrm{HZ}$ BURSTS-1/sec.
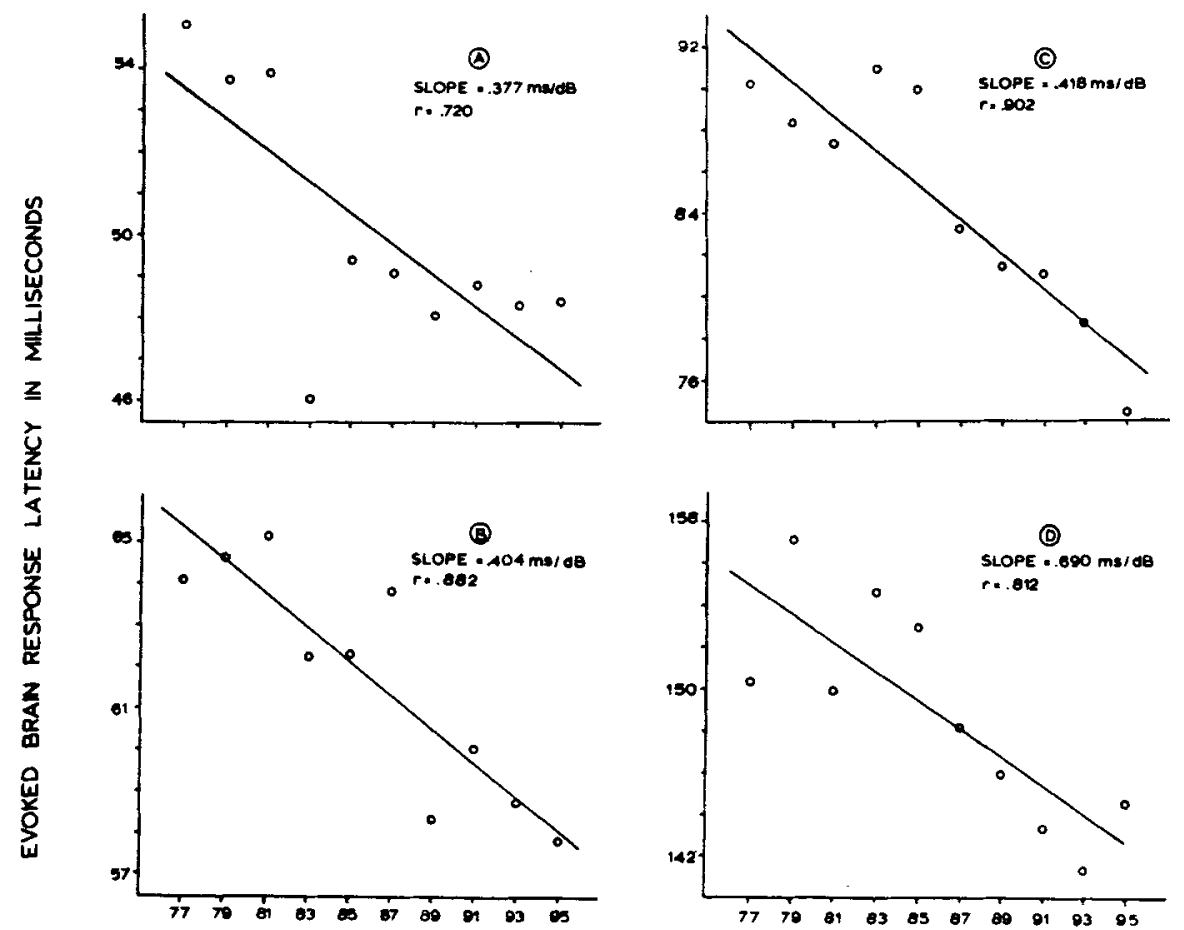

SOUND PRESSURE LEVEL IN DECIBELS

Fig. 7. Evoked brain response latency-intensity functions for $S$ J.N. The regression lines shown were fit to the mean data points using the method of least squares. 
power law mechanisms is premature. The demonstration of linear relationships between auditory EBR amplitude measures and stimulus intensity in no sense conflicts with various studies demonstrating that novelty, attention, drugs, and other factors may affect EBR measures. In a real sense, we have attempted to cancel out such effects by using methodological strategies involving precise stimulus control, large EEG samples, and repeated testing of individual $S$ s on different days. Caution should be exercised, however, in the interpretation of studies indicating EBR changes unrelated to changes in the physical property of the stimulus. Many of these studies may reflect poor stimulus control rather than the implied changes in psychological or physiological state.

\section{REFERENCES}

BUCHSBAUM, M. Neural events and psychophysical law. Science, 1971, 172. 502.

DAVIS, H. Auditory responses evoked in the human cortex. In A. V. S. DeReuck and J. Knight (Eds.), Hearing mechanisms in vertebrates. London: Churchill, 1968. Pp. 259-268.

DAVIS, H., BOWERS, C.. \& HIRSH, S. K. Relations of the human vertex potential to acoustic input: Loudness and masking. Journal of the Acoustical Society of America, 1967, 43, 431-438.

DONCHIN, E., \& SUTTON, $S$. The "psychological significance" of evoked responses: A comment on Clark, Butler, and Rosner. Communications in Behavioral Biology, 1970,5, Part A, 1-5.

JASPER, H. H. The ten twenty electrode system of the international federation. Electroencephalography \& Clinical Neurophysiology, 1958, 10, 371-375.

PRICE, L. L. Evoked response audiometry: Some considerations. Joumal of Speech 4 Hearing Disorders, 1969, 34, 137-141. RAPIN, I., SCHIMMEL, H., TOURK, L. M.. KRASNEGOR, N. A. " POLLAK, C. Evolsed responses to clicks and tones of varying intensity in waking adults. Electroencephal ography \& Clinical Neurophysiology, 1966, 21, 335-344.

ROTHMAN, H. H. Effects of high frequencies and intersubject variability on the auditory-evoked cortical response. Joumal of the Acoustical Society of America, 1969, 47, 569-573.

SCHARF, B. FISHKEN, D. Binaural summation of loudness reconsidered. Journal of Experimental Psychology, $1970,86,374-379$.
SHEPHERD, D. C. Individual specificity of the late components of averaged electroencephatic responses (AERS) evoked by repeated clicks. Journal of Speech \& Hearing Research, 1969, 12 , 762-777.

SIEGFRIED, J. B., TEPAS, D. I., SPERLING, H. G., \& HISS, R. H. Evoked brain potential correlates of psychophysical responses: Heterochromatic hicker photometry. Science, 1965, 149, 321-323.

VAUGHAN, H, G JR \& RITTER, W. The sources of auditory evoked responses recorded from the human scalp. Electroencephalography \& Clinical Neurophy siology, 1970, 28, 360-367.

\section{NOTE}

1. We have used the letters $A, B, C$, and $D$ to identify the components measured to avoid nomenclature difficulties which might arise in the description of related studies which we are conducting using paired-stimuli and cross-modality waveforms. $B, C$, and $D$ are frequently referred to in the literature as $P_{1}, N_{1}$, and $P_{2}$, respectively. It should be noted. however, that the $P$ and $N$ nomenclature is not consistently used in the literature and is more appropriate with reference to monopolar waveforms.

(Received for publication September 10 , 1971.) 